\title{
VIBRATION ANALYSIS OF SHELL AND TUBE HEAT EXCHANGER BY CHANGING BAFFLE SPACING USING CHEMCAD
}

Sameer M. Wagh ${ }^{1}$, Divya P. Barai ${ }^{2} \&$ Megha H. Talwekar ${ }^{3}$

Abstract- Shell and tube heat exchangers are used extensively throughout the process industry. Vibration of tubes in heat exchangers is an important limiting factor in heat exchanger operation. One of the design specification on which vibration is dependent is the unsupported tube length which is a function of baffle spacing. In this paper, the vibration analysis for the shell and tube heat exchanger is carried out at different values of baffle spacing using CHEMCAD. The change in the values of various vibration mechanisms is noted by changing the baffle spacing of a shell with single segmental baffles at $0.072 \mathrm{~m}, 0.076 \mathrm{~m}$ and $0.08 \mathrm{~m}$. The changes in the cross-flow velocity, critical velocity, natural frequency, vortex shedding frequency and turbulent buffeting frequency throughout the length of tube of the heat exchanger are studied.

Keywords: Vibration analysis, shell and tube heat exchanger, CHEMCAD, baffle spacing

\section{INTRODUCTION}

Vibration is a mechanical phenomenon. Vibration motions create unwanted sounds and so are typically unwanted. Due to vibration wastage of energy in a mechanical operation takes place. Vibration becomes a problem in heat exchangers when the intensity increases to the point that it causes some part of the exchanger to fail mechanically, upsets the process conditions, or creates a condition that endangers those who work in that area. Prolonged tube vibration with large amplitudes leads to leakage between the shell side and tube side fluids due to the mechanical failure of tubes. Tubes being the most flexible part of a heat exchanger are vulnerable to flow-induced vibration caused by the flow of fluid past them. Danger of failure arises when the frequency of the tube vibration becomes appreciably high. So, careful designs are made to minimize unwanted vibrations. Vibration Analysis (VA) in any industry aims to detect equipment faults. The shell and tube heat exchangers find their applications in a many sectors. The unsupported tube span in an STHE has major impact on the various vibration mechanisms[1]. The flow-induced vibration analysis of a shell and tube heat exchanger is an integral element of its thermal design.

Most sophisticated thermal design software packages carry out vibration analysis as a routine ingredient of thermal design. A vibration analysis of shell and tube heat exchanger by using HTRI software is presented by Patel [2]. A simplified approach to optimize the design of shell tube heat exchanger by flow-induced vibration analysis is presented by using HTRI software with Horizontal Multi-pass Flow shell with no tubes in window segmental baffles by Gawande et al. [3]. Wagh et al [4] has compared the vibration parameters in a STHE for single segmental baffles and no tubes in window baffles and concluded that their values are more in case of no tubes in window baffles using CHEMCAD.CHEMCAD is a software which is capable of modeling continuous, batch and semi-batch processes. The following flow-induced vibration mechanisms are considered by CHEMCAD to investigate the mechanical stability of a heat exchanger.

Natural frequency: Natural frequency is the frequency at which a system tends to oscillate in the absence of any driving force. It is the frequency at which the tubes vibrate. One of the variables that affect the natural frequencies is the length of the unsupported spans. Most of the heat exchangers have multiple baffle supports and varied individual unsupported spans. The natural frequency of the heat exchanger is an essential step in estimating its potential for its flow induced vibration failure. One should find the natural frequency of vibration of the tubes to study the tube vibrations. Calculation of the natural frequency of the heat exchanger is an essential step in estimating its potential for its flow induced vibration failure[5]. Vortex Shedding Frequency: When a fluid flows across a single tube, it produces a series of vortices in the downstream wake due to the separation of flow alternately from opposite sides of the tube. This alternate shedding of vortices produces alternating forces, the frequency of which varies directly with the velocity of flow. For a given arrangement and tube size, the frequency of the vortex shedding for non-vibrating tubes increases as the velocity increases. The vortex shedding can excite tube vibration when it matches the natural frequency of the tubes. The movement of the tube organizes the separation of the vortices leaving the vibrating tube[6]. Heat exchangers are recommended to be designed so that the natural frequency of the tubes is always greater than the frequency of the vortex shedding. Turbulent Buffeting Frequency: Turbulence is generated when shell side fluid flow through tube bundle. When basic frequency of turbulence pulsating is proximal or equal to natural frequency of tube, fierce vibration will take place. Turbulent buffeting is defined as the fluctuating forces acting on tubes due to extremely turbulent flow on shell side of the gas/liquid. This turbulence buffets the tubes which selectively extracts energy from the turbulence at their natural frequency. So, there is greater impact of the velocity of the flowing fluid on turbulent buffeting frequency[6].

\footnotetext{
${ }^{1}$ Laxminarayan Institute of Technology, Rashtrasant Tukadoji Maharaj Nagpur University, Nagpur, India

${ }^{2}$ Laxminarayan Institute of Technology, Rashtrasant Tukadoji Maharaj Nagpur University, Nagpur, India

${ }^{3}$ Laxminarayan Institute of Technology, Rashtrasant Tukadoji Maharaj Nagpur University, Nagpur, India
} 
Considering the cross-flow velocity and the critical velocity during the study of vibration becomes important due to the constant changes in magnitude and direction of the shell side fluid velocity.

Cross-flow velocity: The definition for cross-flow velocity usually considered when it comes to flow-induced vibration is based on the minimum flow area through a tube row perpendicular to the primary direction of flow. For an ideal tube bank the selected velocity is well defined. For a shell and tube exchanger the interpretation of cross flow velocity is uncertain, as the number of tubes in each row varies from baffle tip to baffle tip. In order to be consistent, the cross flow velocity for shell and tube heat exchanger vibration prediction will be based on an integrated average area between the maximum and minimum number of tubes in the rows between baffle tips, on the gaps between adjacent tubes in a tube row and on the cross flow fraction of the total flow. Critical Velocity: The flow velocity which equalizes hydrodynamic exciting and damping forces and gives rise to hydro elastic vibrations is known as the critical velocity. In simple words, the critical flow velocity for a tube span is the minimum cross flow velocity at which that span may vibrate with unacceptably large amplitudes. The cross flow velocity should always be less than critical flow velocity. There are many design aspects of the exchanger on which the critical velocity of the shell side depends. In this proposed work, vibration analysis of shell and tube heat exchanger is carried out by changing baffle spacing for the first time. Along with the vibration mechanisms considered, a study of cross-flow velocity and critical velocity is done by using CHEMCAD software. This work is carry out in CHEMCAD which uses CC-THERM for the study of heat exchangers.

\section{PROCEDURE}

The baffle spacing which is the distance between the two adjacent baffles considered for the study is changed by using the values of $0.072 \mathrm{~m}, 0.076 \mathrm{~m}$ and $0.08 \mathrm{~m}$. Changes in the values of vibration mechanisms of natural frequency, vortex shedding frequency and turbulent buffeting frequency at inlet, center and outlet parts of tube are noted from the results given by CC-THERM. Also, values of cross flow velocity and critical velocity are noted from the same results. Graphs are drawn depicting the vibration in the shell side throughout the length of the heat exchanger.

\section{RESULTS AND DISCUSSIONS}

The results of the comparison of the vibration analysis of single segmental baffles having different spacing indicated the results as shown in Table -1 .

Table -1 Vibration analysis of shell and tube heat exchanger by changing baffle spacing

\begin{tabular}{|l|l|l|l|l|}
\hline Types of Baffles & Parameters & Inlet & Center & Outlet \\
\hline \multirow{3}{*}{$\begin{array}{l}\text { Single } \\
\text { Segmental }\end{array}$} & Cross-Flow Velocity (m/sec) & 0.1632 & 0.3436 & 0.1632 \\
\cline { 2 - 5 } & Critical Velocity $(\mathrm{m} / \mathrm{sec})$ & 42.25 & 56.37 & 42.02 \\
\cline { 2 - 5 } & Natural frequency $(\mathrm{cycles} / \mathrm{sec})$ & 1396.93 & 1980.47 & 1393.02 \\
\cline { 2 - 5 } & Vortex Shedding Frequency $(\mathrm{cycles} / \mathrm{sec})$ & 4.63 & 9.75 & 4.63 \\
\cline { 2 - 5 } & Turbulent Buffeting Frequency (cycles/sec) & 2.73 & 5.75 & 2.73 \\
\hline \multirow{4}{*}{$\begin{array}{l}\text { No Tubes } \\
\text { Windows }\end{array}$} & Cross-Flow Velocity (m/sec) & 0.1755 & 0.3695 & 0.1755 \\
\cline { 2 - 5 } & Critical Velocity $(\mathrm{m} / \mathrm{sec})$ & 83.44 & 189.75 & 83.10 \\
\cline { 2 - 5 } & Natural frequency (cycles/sec) & 3170.99 & 7923.78 & 3164.00 \\
\cline { 2 - 5 } & Vortex Shedding Frequency (cycles/sec) & 4.98 & 10.49 & 4.98 \\
\cline { 2 - 5 } & Turbulent Buffeting Frequency (cycles/sec) & 2.9389 & 6.1885 & 2.9389 \\
\hline
\end{tabular}

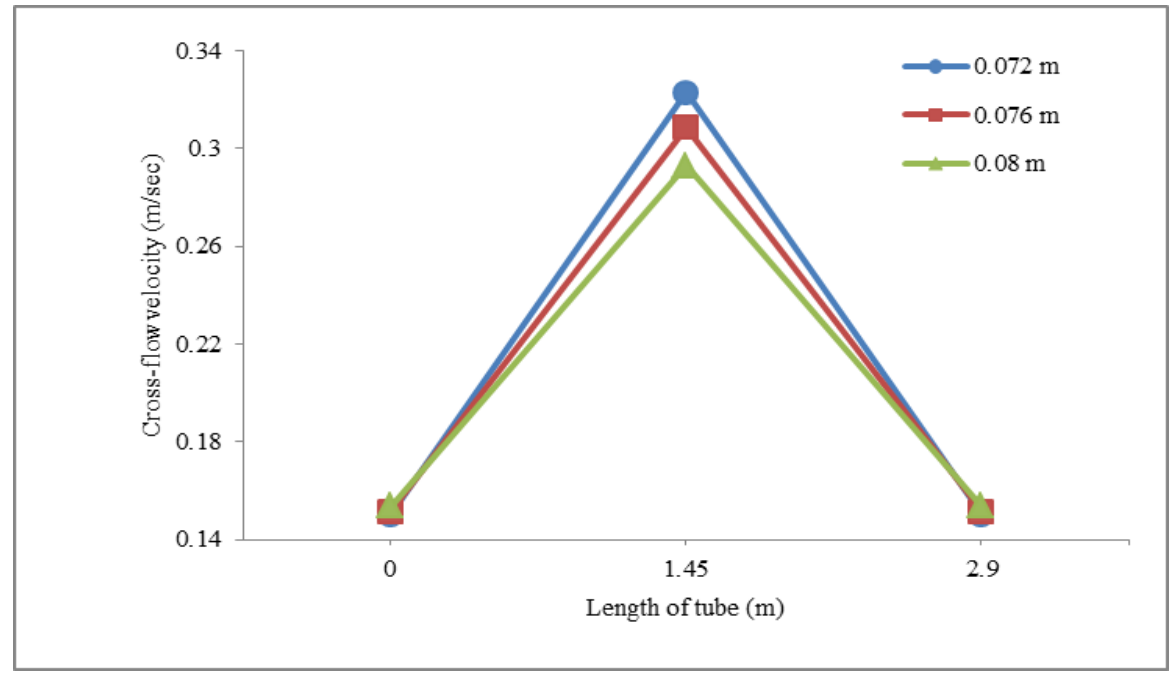

Figure -1 Cross-flow velocity vs length of tube 


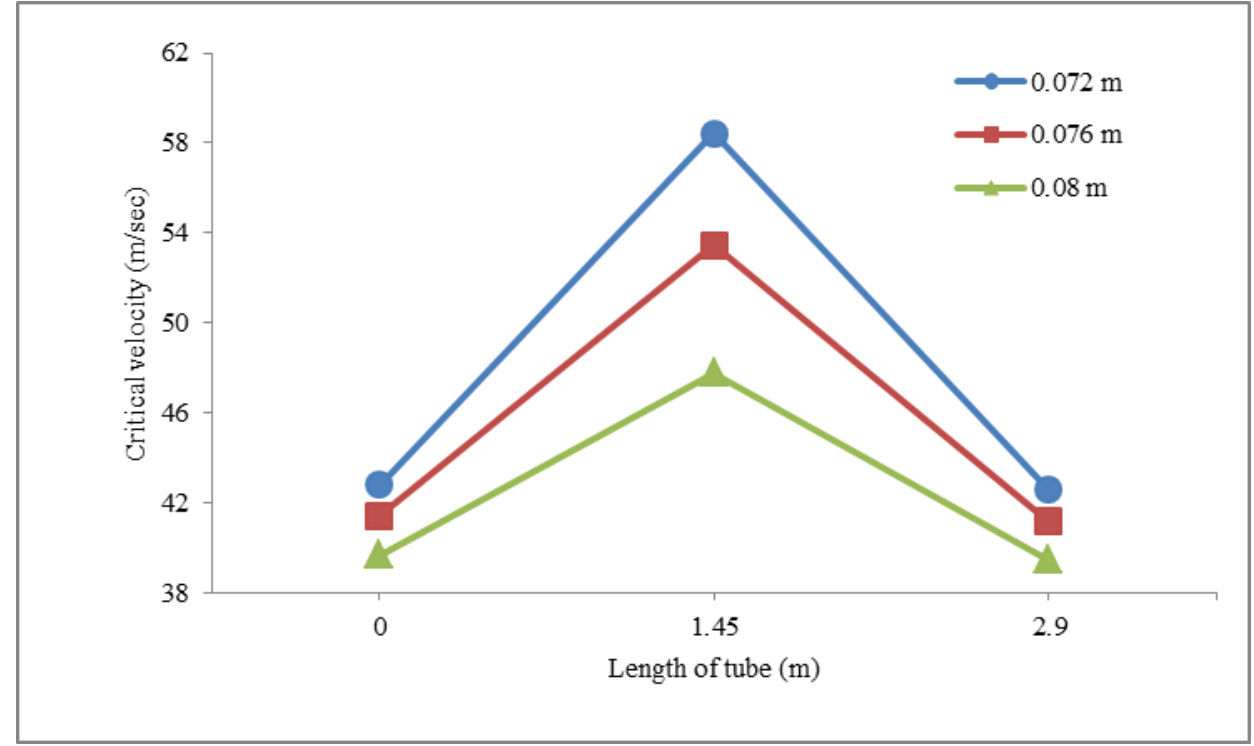

Figure -2 Critical velocity vs length of tube

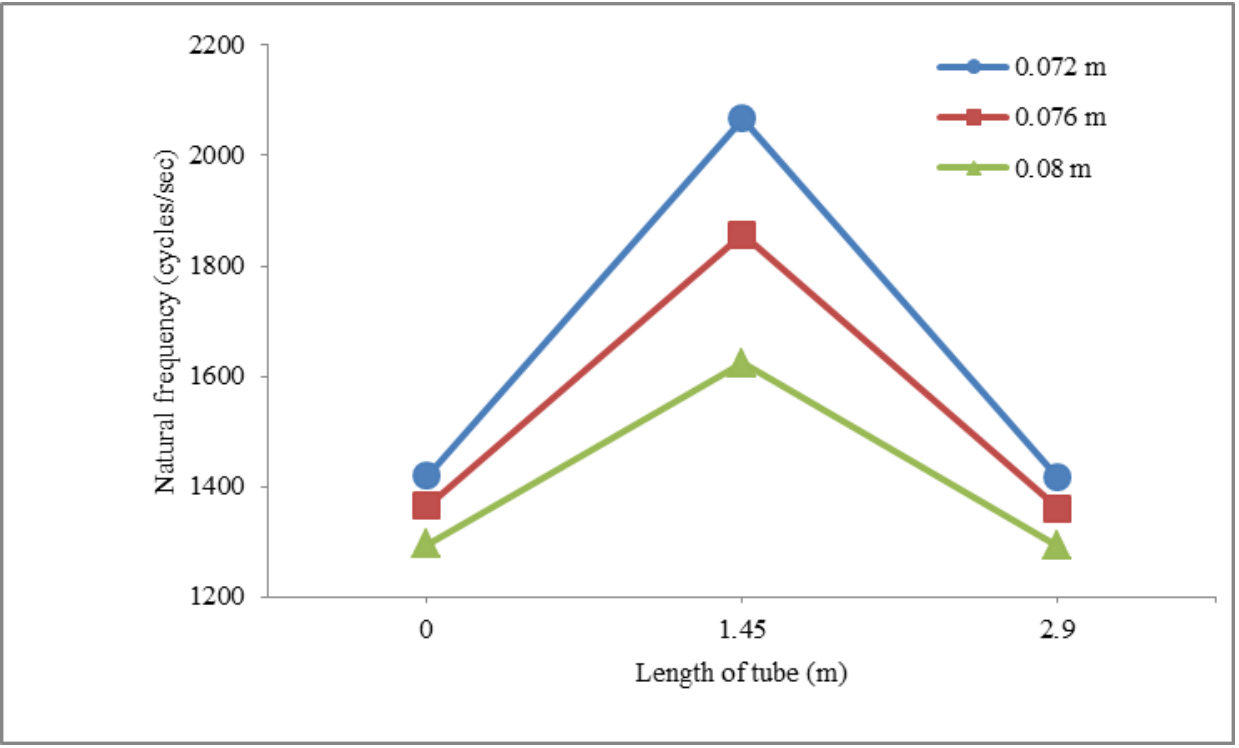

Figure -3 Natural frequency vs length of tube

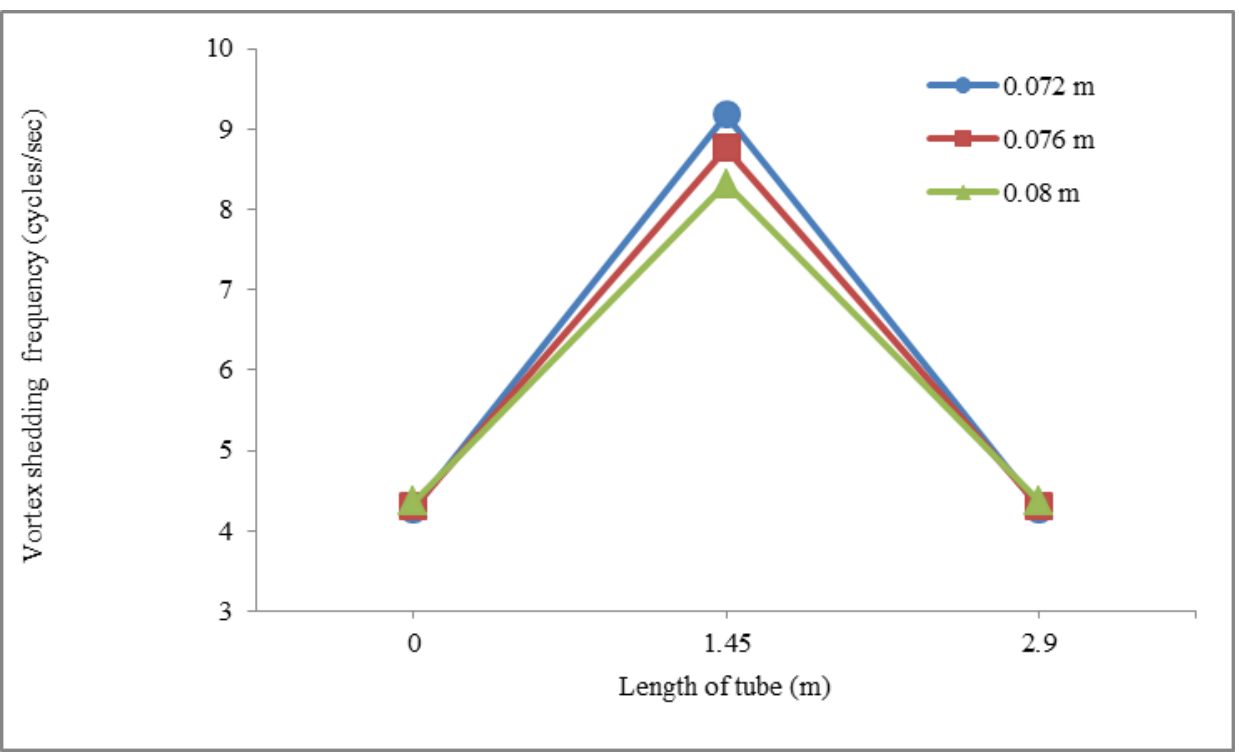

Figure -4 Vortex shedding frequency vs length of tube 


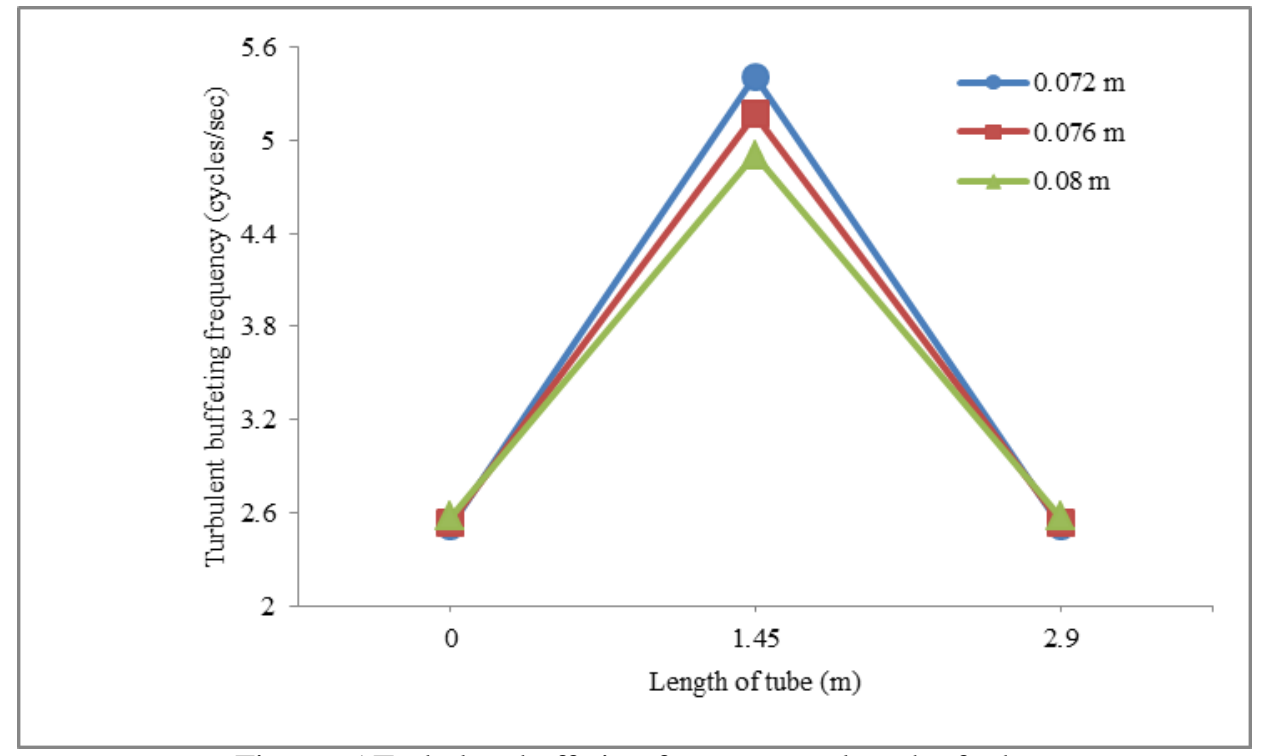

Figure -5 Turbulent buffeting frequency vs length of tube

Figure -1 depicts the cross-flow velocity against the length of tube at different values of baffle spacing. It was observed that the cross-flow velocity increases from inlet to center and then decreases as it approaches to outlet of tube. Also, it was found that as the baffle spacing decreases, the cross-flow velocity slightly increases. The inlet and outlet spacing of the baffle is more and hence cross-flow area for the shell side fluid is more and hence the cross-flow velocity is less at the inlet and outlet. As the fluid approaches the center, baffle spacing is uniform, and therefore there is a uniform increase in the cross flow velocity. After the center, the clearance between the bundle and the shell, tube-to-baffle hole annular clearances reduce the net flow rate of the shell side fluid in cross flow. The reduction in mass flow rate in the cross-flow direction of the shell leads to a decrease in the cross-flow velocity through the length of the heat exchanger from center towards the exit. A decrease in the baffle spacing of the entire heat exchanger decreases the cross-flow area. Thus there is an increase in the cross-flow velocity as the baffle spacing is reduced.

Figure -2 indicates the critical velocity against the length of tube at different values of baffle spacing. It was observed that the critical velocity increases from inlet to center and then decreases as it approaches to outlet of tube. Also it was found that, with the decrease in baffle spacing the critical velocity increases. The vibration damping in shell at the inlet and outlet is lesser than that at the center due to higher inlet and outlet baffle spacing. Thus, the critical velocity at the inlet and outlet of the shell is lesser than the critical velocity of the shell at the center. Vibration damping in shells having less baffle spacing is more due to increased number of baffles. Thus, as the baffle spacing decreases, the critical velocity of the shell increases.

Figure - 3 depicts the natural frequency against the length of tube at different values of baffle spacing. It was observed that the natural frequency increases from inlet to center and then decreases as it approaches to outlet of tube. It was also found that as the baffle spacing decreases, the natural frequency increases. The decrease in the baffle spacing in a shell decreases the tube unsupported length. The natural frequency of the tube is inversely proportional to the unsupported tube length. The inlet and outlet baffle spacing is more than the baffle spacing at the center of the shell. This makes the natural frequency of the shell to be less at the inlet and outlet and to be more at the center. Also, the unsupported tube length in shell with less baffle spacing is less, and so the natural frequency is also more.

Figure -4 and Figure -5 depicts the vortex shedding frequency and turbulent buffeting frequency against the length of tube, respectively at different values of baffle spacing. It was observed that the turbulent buffeting frequency and vortex shedding frequency increases from inlet to center and then decreases as it approaches to outlet of tube and also, as the baffle spacing decreases, the both mechanism slightly increases. The turbulent buffeting frequency and vortex shedding frequency is directly proportional to the cross-flow velocity of the fluid in the shell. We have already seen how the crossflow velocity varies the shell from inlet to center to the outlet. And so the graph for turbulent buffeting frequency and vortex shedding frequency shows similar characteristics of those of cross-flow velocity. Also, for different baffle spacing, we know how the cross-flow velocity varies, and thus the turbulent buffeting frequency and vortex shedding frequency varies similarly.

\section{CONCLUSIONS}

From the vibration analysis of shell and tube heat exchanger, it is found that the tube length has major impact on crossflow velocity, critical velocity, turbulent buffeting frequency, vortex shedding frequency, tube natural frequency. It is indicated from the results that these vibration mechanisms increase with decrease in the baffle spacing. It is also evident 
from the graphs that these vibration mechanisms increase from the inlet to center and decrease as they approach to the outlet.

\section{REFERENCES}

[1] Thombare, T.R.; Kapatkar, V.N.; Utge, C.G.; Raut, A.M.; Durgawale, H.M.. Flow Induced Vibration Analysis of TEMA J-Type U-Tube Shell and Tube Heat Exchanger. Global Journal of Mechanical Engineering and Computational Sciences 2012, 2, 10-14.

[2] Patel, B.M. Vibration Analysis of AES Type Shell and Tube Heat Exchanger by HTRI Software. International Journal of Advanced Engineering Research and Studies 2013, III/I, 94-97.

[3] Gawande, S.H.; Keste, A.A.; Navale, L.G.; Nandgaonkar, M.R.; Sonawane, V.J.; Ubarhande, U.B. Design Optimization of Shell and Tube Heat Exchanger by Vibration Analysis. Modern Mechanical Engineering 2011, 1, 6-11.

[4] Wagh, S.M.; Barai, D.P.; Talwekar, M.H. Comparative Vibration Analysis of Segmental Baffles with No Tubes In Window Baffles in STHE using CHEMCAD. Asian Journal of Science and Technology 2017, 08, 5361-5364.

[5] Patil, R. V.; Bhutada, S.S.; Katruwar, N. R.; Rai, R. R.; Dhumke, K. N. VibrationalAanalysis of Shell and Tube Type of Heat Exchanger in Accordance with Tubular Exchanger Manufacturer's Association (TEMA) Norms. The International Journal of Engineering and Science 2014, 3 , 59-64.

[6] Schlunder, E.U. Heat Exchanger Design Handbook. VDI-Verlag GmbH 1983, Dusseldorf. 